\title{
Gestaltungstheoretische Überlegungen zu Kompetenzmanagementsystemen
}

\author{
Kompetenzentwicklung stellt für Organisationen und ihre Mitarbeiter einen kritischen \\ Erfolgsfaktor im Wettbewerb dar. Kompetenzmanagementsysteme tragen zu einer \\ systematischen Kompetenzentwicklung in Organisationen bei, indem Prozesse wie \\ Bedarfsanalyse, Maßnahmenplanung, Maßnahmenumsetzung und Evaluation unterstützt \\ werden. Bei der Gestaltung von Kompetenzmanagementsystemen ist jedoch eine Vielzahl \\ technischer und organisatorischer Herausforderungen zu bewältigen. Dieser Beitrag \\ präsentiert systematisch aufgearbeitete Gestaltungsempfehlungen für \\ Kompetenzmanagementsysteme, um diesen Herausforderungen zu begegnen. Die \\ Gestaltungsempfehlungen in den Kategorien Organisation, Anwender, Ressourcen und \\ Kompetenzmanagementsoftware reichen von Aufmerksamkeitsmanagement bis hin zur \\ Aktivierung der Akteure durch organisatorische Strukturen und Werte.
}

DOl 10.1007/s11576-010-0251-1

\begin{abstract}
Der Autor
Dr. Bernd Simon ( $\varangle)$

Institut für Wirtschaftsinformatik und Neue Medien

Wirtschaftsuniversität Wien

1090 Wien

Österreich

bernd.simon@wu.ac.at

Eingegangen: 2009-07-30

Angenommen: 2010-09-03

Angenommen nach sechs Überarbei-

tungen durch Prof. Dr. Buhl.

Online publiziert: 2010-11-03

This article is also available in English via http://www.springerlink.com and http://www.bise-journal.org: Simon B (2010) A Discussion on Competency Management Systems from a Design Theory Perspective. Bus Inf Syst Eng. doi: 10.1007/s12599-010-0134-4.

(C) The Author(s) 2010. Dieser Artikel ist auf Springerlink.com mit Open Access verfügbar
\end{abstract}

\section{Einleitung}

\subsection{Praktische Bedeutung von Kompetenzmanagement in Organisationen}

Für jede Organisation stellen die Kompetenzen ihrer Mitarbeiter einen kritischen
Erfolgsfaktor dar (Devaraj und Babu 2004, S. 63). Insbesondere bei Veränderungen, beispielsweise als Reaktion auf eine Verschlechterung der gesamtwirtschaftlichen Rahmenbedingungen oder bei der Einführung von Managementinnovationen und neuer Produkte, spielt Kompetenzentwicklung eine wesentliche Rolle (Deiser 2009, S. 45-50; Simon et al. 2008, S. 96; Hamel 2007, S. 41). Gleichzeitig lässt sich aus Sicht des Individuums ein zunehmender Kompetenzentwicklungsbedarf aus den veränderten Anforderungen der Arbeitswelt, wie etwa erhöhte Komplexität, Dynamik, Vernetzung und Unsicherheit, ableiten (Erpenbeck und Sauter 2007, S. 7). Organisationen sind deshalb verstärkt gefordert, sich der Entwicklung von Mitarbeiterkompetenzen anzunehmen (Heyse und Ortmann 2008, S. 19-22; Zwick 2004, S. 663).

Die Steuerung von Kompetenzentwicklung wird als Kompetenzmanagement bezeichnet, eine Managementaufgabe, die in den Bereich der Personalentwicklung fällt. In der betrieblichen Praxis fasst die Personalentwicklung sämtliche Maßnahmen zusammen, die der systematischen Entwicklung von Mitarbeiterkompetenzen dienen (Solga et al. 2008, S. 19). Die Personalentwicklung befindet sich in den letzten Jahren in einem Wandel, da verstärkt Aspekte wie Kostenreduktion, Ausrichtung auf den Unternehmenserfolg und gezielte Talentförderung in den Mittelpunkt gerückt sind, während gleichzeitig die strategische Bedeutung der Personalentwicklung von den Führungskräften zunehmend erkannt wird (Faltin und Zimmermann 2009, S. 6, 7; Heyse und Ortmann 2008, S. 20, 21).

Informationssystemen kommt in solch einem Umfeld eine tragende Rolle zu. Informationssysteme werden als Systeme verstanden, die Informationen sammeln, verarbeiten, speichern und verteilen, um Entscheidungsfindungsprozesse zu unterstützen (Laudon und Laudon 2005, S. 8). Einem organisationstheoretischen Verständnis von Informationssystemen folgend, bezeichnen Informationssysteme soziotechnische Systeme, die nicht nur Informations- und Kommunikationstechnologie (Hardware und Software), sondern auch menschliche Komponenten (Anwender mit bestimmten Kompetenzen) und die organisatorische Umgebung des Systems (Strukturen und Prozesse) umfassen (Picot und Baumann 2009, S. 72). Der vorliegende Beitrag befasst sich mit Informationssystemen zur Unterstützung von Kompetenzmanagement, die hier als Kompetenzmanagementsysteme (KMS) bezeichnet werden.

Die für den Aufbau von KMS am Markt verfügbaren Softwareprodukte haben sich in den letzten Jahren stark weiterentwickelt. Kompetenzmanagementsoftware stellt mittlerweile umfangreiche Funktionen zur Verfügung, um Kompetenzentwicklungsbedarf $\mathrm{zu}$ analysieren, Kompetenzentwicklungsprozesse $\mathrm{zu}$ gestalten und umzusetzen 
sowie $\mathrm{zu}$ evaluieren (Erpenbeck und Sauter 2007, S. 273-276). Eine besondere Ausprägung von Kompetenzmanagementsoftware stellt neuerdings Talentmanagementsoftware dar. Talentmanagementsoftware ergänzt Kompetenzmanagementsoftware um Funktionen zur Identifikation kritischer Rollen und Aufgaben und unterstützt bei der Bereitstellung von erfolgskritischen Kompetenzentwicklungsmaßnahmen für ausgewählte Zielgruppen, sog. Talente (Heyse und Ortmann 2008, S. 11).

Die Einführung von Kompetenzmanagementsoftware und der damit verbundene Aufbau eines KMS stellt Unternehmen jedoch vor besondere Herausforderungen, zum Beispiel:

- Wie kann es gelingen, dass alle für die Kompetenzentwicklung wesentlichen Personen wie Führungskräfte, Lernende oder Personalentwickler ausreichend involviert sind und ihre Entscheidungsfindungen entsprechend unterstützt werden?

- Wie können KMS gestaltet werden, um die Einbeziehung erfolgskritischer, externer Ressourcen wie Vortragende oder Lernmaterialien in allen wesentlichen Prozessen einfach $\mathrm{zu}$ ermöglichen?

- Wie kann die Steuerung von Kompetenzentwicklung intensiviert und verbessert werden?

- Wie können insbesondere Klein- und Mittelbetriebe, in denen keine stark ausgeprägten Strukturen für die Unterstützung von Kompetenzentwicklung vorhanden sind, möglichst einfach $\mathrm{Zu}$ gang zu Software, Methoden und Inhalten erlangen?

Dieser Beitrag widmet sich diesen und ähnlichen Fragen, indem Gestaltungsempfehlungen für KMS erarbeitet werden.

\subsection{Forschungsfrage und wissenschaftliche Bedeutung}

Die Personalwirtschaft gilt generell als Pionierbereich für den Einsatz betrieblicher Anwendungssoftware und damit der Wirtschaftsinformatik (Strohmeier 2000, S. 90). Dies trifft jedoch nicht auf den in den letzten Jahren stark veränderten Bereich des Kompetenzmanagements zu. Obwohl Kompetenzmanagement sowohl aus Sicht der Praxis als auch aus organisationstheoretischer Sicht im letzten Jahrzehnt zunehmend an Bedeutung gewinnt, wurde dieser Bereich von der Wirtschaftsinformatik und verwandten Disziplinen weitgehend vernachlässigt (Hasenkamp und Stahlknecht 2009, S. 6; Lindgren et al. 2004, S. 436; Strohmeier 2000, S. 90).

In der amerikanischen Schwesterdisziplin „Information Systems“ entstanden in den vergangen Jahrzehnten vor allem behavioristische Modelle zur Erfolgsmessung von Informationssystemen (DeLone und McLean 2003) oder der Technologieakzeptanz (Venkatesh 2000). Diese Modelle dienen vor allem dazu, im Nachhinein den Erfolg von Informationssystemen zu erklären und nennen da$\mathrm{zu}$ einige wenige Einflussgrößen wie etwa den wahrgenommenen Nutzen hinsichtlich der eigenen Leistung oder die wahrgenommene Einfachheit der Bedienung (Davis 1989, S. 320). Gestaltungsempfehlungen für Informationssysteme, die darüber hinaus auch noch die Besonderheiten von KMS berücksichtigen, lassen sich aus diesen Modellen jedoch nur bedingt ableiten. Viel mehr fokussieren diese Beiträge auf die Entwicklung von möglichst allgemein gültigen Erklärungsmodellen.

Aus organisationstheoretischer Sicht kann die Arbeit der ressourcenbasierten Führungstheorie zugeordnet werden. Diese wird vor allem dazu herangezogen, existierende realwirtschaftliche Phänomene zu erklären (Erklärungsfunktion). Die Gestaltungsfunktion der Theorie wurde bislang jedoch noch kaum erforscht. „Zukünftig sollten [deshalb] ressourcenorientierte Arbeiten stärker darauf abzielen, nicht nur unternehmerische Ressourcen, Potenziale und Kernkompetenzen aufzulisten, sondern zu zeigen, wie derartige Rentenstifter geschaffen und geschützt werden können (Macharzina und Wolf 2008, S. 69)“. Diese Arbeit versucht hier anzusetzen, indem Gestaltungsempfehlungen für KMS erarbeitet werden. Damit soll aufgezeigt werden, wie solche Informationssysteme effektiv gestaltet werden können, um zu einer unternehmensrelevanten Steigerung von individuellen Kompetenzen und damit auch zu einer Steigerung der Wettbewerbsfähigkeit beizutragen.

Dieser Beitrag möchte an die angeführte Forschung anschließen und gestaltungstheoretische Überlegungen für die Entwicklung von KMS anstellen. Dem Paradigma der Designwissenschaften (engl. Design Science) folgend wurden dazu mithilfe von begründenden betriebswirtschaftlichen Theorien (sog. Kernel Theories) Grundanforderungen für KMS abgeleitet und in einem Prototyp umgesetzt. Der Prototyp wurde anschließend Anforderungen von Organisationen gegenübergestellt und eine anschließende Einführung im Sinne einer Aktionsforschung mit qualitativen Interviews reflektierend begleitet. Aus den Interviews ließen sich mittels Inhaltsanalyse Erfolgsfaktoren für die Gestaltung von KMS ableiten.

Der Beitrag ist wie folgt strukturiert: Abschn. 2 beschreibt das methodische Vorgehen des Forschungsprojekts. Abschn. 3 arbeitet relevante organisations- und weiterbildungstheoretische Grundlagen der Forschungsfrage auf. Abschn. 4 widmet sich dem zentralen Konzept dieser Arbeit, dem Kompetenzmanagementsystem, und definiert theoriegeleitet Anforderungen. Abschn. 5 präsentiert Prototyp und Evaluationsergebnisse sowie Limitationen der Untersuchung. Die Forschungsergebnisse werden in Abschn. 6 nochmals zusammengefasst und aus wissenschaftlicher Sicht diskutiert.

\section{Epistemologische Positionierung und Forschungsmethode}

\subsection{Epistemologische Positionierung}

Diese Arbeit ist dem Paradigma der Designwissenschaften zuzuordnen. Angewandt auf die Wirtschaftsinformatik, werden in diesem Wissenschaftsparadigma Artefakte entwickelt und evaluiert, um zuvor identifizierte Problemstellungen im Zusammenspiel von Technik und sozialen Systemen zu lösen (Gregor und Jones 2007, S. 314; Hevner et al. 2004, S. 77).

Gregor und Jones (2007, S. 321) unterscheiden informationstechnologierelevante Artefakte wie Prototypen von informationssystem-relevanten Artefakten wie Gestaltungsprinzipien für Informationssysteme, wobei Prototypen zur Entwicklung von Letzteren beitragen können (Hevner et al. 2004, S. 77). Diese Rolle kommt dem Prototyp auch in dieser Arbeit zu.

Ziel des Forschungsprojekts ist die Erarbeitung einer Menge an relevanten Gestaltungsempfehlungen für die Entwicklung von KMS. Gestaltungsprinzipien für Informationssysteme umfassen beispielsweise Anforderungen an Anwender und Systemfunktionen sowie Prinzipien für eine effektive Entwicklung bzw. einem effektiven Betrieb (Markus et al. 2002, S. 181). 
Der Erkenntnisfortschritt manifestiert sich dabei vor allem in den überprüfbaren Propositionen, die in dieser Arbeit durch eine prototypengetriebene Evaluation mit anschließender Inhaltsanalyse erarbeitet wurden.

\subsection{Forschungsmethode}

Um überprüfbare Propositionen hinsichtlich einer erfolgreichen Gestaltung von KMS zu erarbeiten, lag der Schwerpunkt des Forschungsprojekts in der Evaluationsphase. Diese orientierte sich an den Prinzipien der Aktionsforschung (Davison et al. 2004, S. 69):

1. Prinzip des Übereinkommens zwischen Forscher und Kunden über das (Begleitforschungs-)Projekt,

2. Prinzip des zyklischen Prozesses,

3. Prinzip der theoretischen Fundierung,

4. Prinzip einer angestrebten Veränderung,

5. Prinzip des Lernens durch Reflexion.

Bei der Aktionsforschung handelt es sich um einen sozialwissenschaftlichen Forschungsansatz, der sich aufgrund seiner Anwendungsorientierung gut für Informationssystemforschung und gestaltungswissenschaftliche Forschung eignet (Baskerville und Wood-Harper 1998, S. 90). Das Besondere an der Aktionsforschung besteht in ihrem intervenierenden Ansatz, der sowohl in wissenschaftlicher als auch in praktischer Hinsicht eine relevante Problemstellung adressiert (Easterby-Smith et al. 2008, S. 165) und im Forscher-KundenÜbereinkommen dokumentiert wird.

Der in diesem Projekt für die Intervention vorbereitete Prototyp bietet vor allem für mittelständische Unternehmen eine Alternative $\mathrm{zu}$ innerbetrieblicher Standardsoftware, wobei zwei Anwendungsbereiche unterschieden werden: Der Anwendungsbereich „innerbetriebliche Kompetenzentwicklung“ zielt auf die innerbetrieblich gesteuerte Personalentwicklung $\mathrm{ab}$, weshalb hier mit Vertretern von Personalabteilungen und mit Führungskräften gesprochen wurde. Der zweite Anwendungsbereich richtet sich an Beratungsunternehmen, die mit dem Portal eine Lösung für die Durchführung von Kompetenzentwicklungsprojekten bei ihren Kunden vorfinden. Infolgedessen wurde der Prototyp deshalb auch Weiterbildungsanbietern vorgestellt. Primäre Anwender in diesem Szenario sind externe Berater und Trainer, die Analysen, Interventionen oder

Tab. 1 Evaluation: Involvierte Entscheidungsträger und Interviewanzahl

\begin{tabular}{|c|c|c|c|c|c|c|}
\hline $\begin{array}{l}\text { Unter- } \\
\text { nehmen }\end{array}$ & $\begin{array}{l}\text { Personal- } \\
\text { leiter }\end{array}$ & $\begin{array}{l}\text { Interne } \\
\text { Wissens- } \\
\text { manager }\end{array}$ & $\begin{array}{l}\text { Führungs- } \\
\text { kraft }\end{array}$ & $\begin{array}{l}\text { Externer Wei- } \\
\text { terbildungs- } \\
\text { dienstleister }\end{array}$ & $\begin{array}{l}\text { Interner Wei- } \\
\text { terbildungs- } \\
\text { dienstleister }\end{array}$ & $\begin{array}{l}\text { Software- } \\
\text { einführung }\end{array}$ \\
\hline A & 3 & & & & & $\mathrm{Ja}$ \\
\hline B & 3 & & & & & $\mathrm{Ja}$ \\
\hline $\mathrm{C}$ & 2 & & & & & $\mathrm{Ja}$ \\
\hline $\mathrm{D}$ & & & & 2 & & $\mathrm{Ja}$ \\
\hline E & & & & 3 & & $\mathrm{Ja}$ \\
\hline $\mathrm{F}$ & & & 1 & & & $\mathrm{Ja}$ \\
\hline G & & & 1 & & & Nein \\
\hline $\mathrm{H}$ & & & 1 & & & Nein \\
\hline I & & & 1 & & & Nein \\
\hline $\mathrm{J}$ & & & & & 1 & Nein \\
\hline $\mathrm{K}$ & 1 & 1 & & & & Nein \\
\hline $\mathrm{L}$ & 1 & & & & & Nein \\
\hline M & 1 & & & & & Nein \\
\hline $\mathrm{N}$ & 1 & & & & & Nein \\
\hline $\mathrm{O}$ & 1 & & & & & Nein \\
\hline
\end{tabular}

Evaluationen im Auftrag ihrer Kunden durchführen.

Insgesamt bestand im Rahmen des Forschungsprojekts über einen Zeitraum von zwei Jahren zu 55 Unternehmen Kontakt. Von den 55 Unternehmen konnten 15 für eine Prototypenevaluation gewonnen werden. Veränderte wirtschaftliche Rahmenbedingungen, aber auch unterschiedlich stark ausgeprägtes Interesse an der Problemstellung resultierten in einem unterschiedlich starken Engagement und damit in auch - im Sinne der Aktionsforschung unterschiedlich stark ausgestalteten Veränderungen. Bei sechs der 15 Unternehmen kam es zu einer Einführung und damit einer „tiefen“ Intervention. Bei weiteren neun Unternehmungen fanden ein Pilotbetrieb und eine Demonstration mit ausgewählten Anwendern statt.

Die Einführung bzw. Demonstration wurde von Interviews begleitet, die der gemeinsamen Reflexion von Forscher und Praktiker dienten. Je nach dem Grad der Involvierung des Unternehmens wurden ein bis drei Interviews mit unterschiedlichen Funktionsträgern geführt. Tabelle 1 gibt eine Übersicht über die involvierten Stellen und die Anzahl der jeweils durchgeführten Interviews.

Die Interviews waren schwach strukturiert und folgten einem Interviewleitfaden, der sich im Sinne eines qualitativen Vorgehens im Laufe des Projekts von Interview zu Interview weiterentwickelte (Rubin und Rubin 1995, S. 43-48). In den Interviews wurden Informationen $\mathrm{zu}$
Unternehmen und Ansprechpartnern, einem implementierten KMS, einer Bewertung des Status quo, den Einflussfaktoren, die eine erfolgreiche Weiterentwicklung begünstigen, sowie Feedback zum Prototyp erhoben.

Die Auswahl der Unternehmen orientierte sich unter den gegebenen Rahmenbedingungen an der Strategie der maximal möglichen Heterogenität (Patton 2002, S. 234). Als Konsequenz dieser Strategie wurden sowohl Mitarbeiter von Großunternehmen (vor allem zu Anwendungsbereich 1) als auch Mitarbeiter von kleinen Beratungshäusern mit weniger als 50 Mitarbeitern (hier vor allem zu Anwendungsbereich 2) befragt.

Die Interviews wurden transkribiert und im Anschluss inhaltsanalytisch ausgewertet. Es war Ziel der qualitativen Inhaltsanalyse Themen, Muster und Kategorien zu identifizieren, die für das untersuchte Phänomen von Relevanz waren (Easterby-Smith et al. 2008, S. 175). Die dabei angewandte, zusammenfassende Inhaltsanalyse wurde in einem dreistufigen Verfahren umgesetzt (Mayring 2010, S. 59-62):

1. Zusammenfassende Paraphrasierung der Interviews,

2. Generalisierung der Paraphrasen,

3. Reduktion durch Selektion, Bündelung, Konstruktion und Integration von Paraphrasen.

Am Ende jeder Reduktionsphase wurde genau überprüft, ob die als Kategoriensystem zusammengestellten neuen Aussagen das Ausgangsmaterial voll- 
ständig repräsentieren. Darüber hinaus wurden in den Reduktionen teilweise auch quantitative Perspektiven auf das Datenmaterial eingearbeitet. Dadurch sollen weitere Hinweise bezüglich der Bedeutung der jeweiligen Gestaltungsempfehlungen gegeben werden. Im Anhang wird das Vorgehen beispielhaft anhand der Gestaltungsempfehlung „Benchmark-Werte "illustriert.

Ziel dieses Vorgehens war eine analytische Generalisierung, indem Propositionen herausgearbeitet wurden, die von allgemeinerer Bedeutung sind (Mayring 2010, S. 320). Die generierten Propositionen sind als theorienentwickelnd (vs. theorienprüfend) zu verstehen (Atteslander 2008, S. 200)

\section{Organisations- und weiterbildungstheoretische Grundlagen}

\subsection{Kompetenzen von Organisationen und ihrer Mitglieder}

Die ressourcenbasierte Führungstheorie geht davon aus, dass der Erfolg bzw. Misserfolg von Unternehmen vorrangig durch ihre Kernkompetenzen bestimmt wird (Macharzina und Wolf 2008, S. 65). Unter Kernkompetenzen werden die über Lernprozesse entwickelten Fähigkeiten zur Koordination der im Unternehmen eingesetzten Ressourcen verstanden (Prahalad und Hamel 1990, S. 82). Diese Fähigkeiten finden sich „eingebettet“ in den Kompetenzen der Organisationsmitglieder und in der Organisation selbst in Form von Prozessen und Strukturen (Argote et al. 2003, S. 579).

Die für die Personalentwicklung wesentliche Disziplin der Organisationspsychologie verleiht dem Kompetenzbegriff noch eine andere Bedeutung. Aus organisationspsychologischer Perspektive bezeichnen Kompetenzen nämlich die persönlichen Fähigkeiten eines Individuums (Dispositionen) zur Selbstorganisation (Erpenbeck und Sauter 2007, S. 65). Dabei wird Qualifikation, im Sinne von individueller Handlungsfähigkeit, um Aspekte wie Regeln, Normen, Werte und Einstellungen ergänzt, wobei gleichzeitig auch die Selbstorganisation im Vordergrund steht (Erpenbeck und Sauter 2007, S. 69). Die Entwicklung von Regeln, Normen und Werten innerhalb einer Organisation stellt dabei eine wesentliche Voraussetzung dar (Heyse und Ortmann 2008, S. 14)
Die Herausbildung einer unternehmerischen Kernkompetenz erfordert somit die Entwicklung von Qualifikationen beim eingesetzten Personal, wobei aus organisationspsychologischer Sicht dazu auch eine Entwicklung von Regeln, Normen und Werten erforderlich ist. Die Qualifizierung von Mitarbeitern wird um ein „Wertlernen“ ergänzt (Erpenbeck und Sauter 2007, S. 68, 69). Im Falle eines aus Unternehmensanforderungen abgeleiteten Qualifizierungsbedarfs und der um Wertlernen erweiterten Wissensvermittlung, inkludiert die Entwicklung von individuellen Mitarbeiterkompetenzen in diesem Sinne auch Aspekte des organisatorischen Lernens (Argote et al. 2003, S. 579). KMS spielen dabei eine besondere Rolle, da diese Prozesse abbilden, die eine solche Kompetenzentwicklung steuern. Sie unterstützen damit Unternehmen, Fähigkeiten zu entwickeln, die tief im Unternehmen verwurzelt sind und nicht oder nur sehr aufwändig extern beschafft werden können (Czerny 2009, S. 23; Macharzina und Wolf 2008, S. 67).

\subsection{Besondere Merkmale von Kompetenzentwicklungsmaßnahmen}

Bei der Bereitstellung von Kompetenzentwicklungsmaßnahmen handelt es sich um Leistungen von besonderer Qualität. Oft ist die Einbindung externer Wissensträger für die Leistungserbringung erfolgskritisch, jedoch stellt dies für das Prozessmanagement bzw. für die Informationssystemgestaltung eine besondere Herausforderung dar.

Aus ökonomischer Sicht lassen sich Leistungen wie die Bereitstellung von Kompetenzentwicklungsmaßnahmen nach dem Grad der zur Leistungserbringung erforderlichen Einbeziehung externer Ressourcen klassifizieren (Engelhart et al. 1993, S. 401). Um eine Ausrichtung der Kompetenzentwicklung an den Anforderungen der Organisation sicherzustellen, ist bei Kompetenzentwicklungsmaßnahmen ein intensives Zusammenwirken von verschiedenen Akteuren inner- und außerhalb der Organisation, wie zum Beispiel Führungskraft, Mitarbeiter, Personalentwicklung, externer Vortragender und externer Qualifizierungsanbieter, erforderlich (Faltin und Zimmermann 2009, S. 6).

Eine Optimierung der Informationsund Kommunikationsprozesse rund um die Einbindung externer Ressourcen hat das Potenzial, entscheidend auf die Leistungsbereitstellung Einfluss zu nehmen (Simon et al. 2008, S. 96; Hertel und Konradt 2004, S. 10-14). Ein durch KMS verbesserter Informationsfluss zwischen Leistungsanbieter und Nachfrager eröffnet Möglichkeiten zur Individualisierung der Dienstleistung - ein wesentlicher Erfolgsfaktor für Kompetenzentwicklungsmaßnahmen (Simon und Treiblmaier 2007, S. 344) - und reduziert das Beschaffungsrisiko.

\section{Grundanforderungen an Kompetenzmanagementsysteme}

Unter Kompetenzmanagementsystemen werden in dieser Arbeit Informationssysteme verstanden, die der Kompetenzentwicklung dienen. Auf Basis der in Abschn. 1 angeführten Definition von Informationssystemen sowie der organisations- und weiterbildungstheoretischen Grundlagen sind Kompetenzmanagementsysteme in dieser Arbeit wie folgt definiert: Kompetenzmanagementsysteme werden als soziotechnische Systeme verstanden, die Kompetenzmanagementsoftware einsetzen, um Anwendern im Kontext ihrer Organisation Kompetenzentwicklung zu ermöglichen und in diesem Zusammenhang Entscheidungsunterstützung hinsichtlich des Einsatzes organisationsexterner und -interner Ressourcen zu leisten.

Um Kompetenzmanagement in diesem Sinne zu betreiben, ist das Erfassen von für Kompetenzentwicklung relevanten Informationen (z. B. Leistungsbeurteilungen, Qualifizierungsbedarfe, Bewertungen von Vortragenden) und die Reaktion auf diese Informationen (z. B. Gestaltung eines individuellen Entwicklungsplans, Bereitstellung eines Weiterbildungsangebots, Förderung von Vortragenden) erforderlich.

Kompetenzentwicklungsprozesse können anhand eines dreiphasigen Modells strukturiert werden (Erpenbeck und Sauter 2007, S. 273-276), ähnlich, wie dies auch Wissensmanagementbeiträge (Probst et al. 2000, S. 30), Bildungscontrollingbeiträge (Seeber 2000, S. 37) sowie Personalentwicklungsbeiträge (Sonntag 2005, S. 25) vorschlagen:

(1) Analyse: Bedarfs- und Zielanalyse zur systematischen Aufarbeitung von Kompetenzentwicklungsbedarfen und -zielen. 
(2) Intervention: Gestaltung und Umsetzung von Kompetenzentwicklungsmaßnahmen mithilfe entsprechend auf- bzw. vorbereiteter Lernund Transferumgebungen.

(3) Evaluation: Bewertung des Kompetenzentwicklungserfolgs.

Zur Erhebung der für die Kompetenzentwicklung wesentlichen Steuerungsinformation kommen in den einzelnen Phasen unterschiedliche Methoden zum Einsatz, auf die nunmehr kurz eingegangen werden soll.

\subsection{Analyse von Kompetenzentwicklungsbedarf}

Die Methoden in der Analysephase können dahingehend differenziert werden, ob sie auf einer Organisationszielanalyse basieren bzw. von der Organisationsstrategie abgeleitet werden, ob eine Aufgaben- und Anforderungsanalyse im Mittelpunkt steht oder ob sie auf individuelle Bedürfnisse fokussieren (Heyse und Ortmann 2008, S. 14; Rosenstiel 2006, S. 109).

Das Mitarbeitergespräch, auch Förder-, Führungs- oder Zielsetzungsgespräch genannt, befasst sich mit dem Organisationsmitglied und dessen Leistung, kann jedoch unter Zuhilfenahme entsprechender Ressourcen auch für eine auf die Organisationsstrategie ausgerichtet Kompetenzmessung eingesetzt werden. Die Methode hat sich in den letzten Jahren als wichtige Aktivität zur Ermittlung von individuellen Kompetenzentwicklungsbedarfen etabliert. Zu den drei Hauptaufgaben des Mitarbeitergesprächs zählen die Bereitstellung einer Rückmeldung zur Mitarbeiterleistung, das Aufzeigen von Entwicklungserfordernissen sowie die Vereinbarung von Zielen (Schuler und Görlich 2006, S. 254).

Beim 360-Grad-Feedback werden organisationsrelevante Kompetenzen von Führungskräften erfasst und bewertet. Die mehrperspektivische Datenerhebung umfasste ursprünglich eine Selbsteinschätzung sowie eine Fremdeinschätzung durch Mitarbeiter, Kollegen und Vorgesetzte und wurde später auch auf andere Akteure wie Trainer, Berater und Kunden erweitert (Edwards und Ewen 2000, S. 42). Das 360-Grad-Feedback wird deshalb auch Rundumbeurteilung genannt. Beim 360-Grad-Feedback kommt dem eingesetzten Fragebogeninstrument eine besondere Bedeutung zu, da dieses unter Voraussetzungen wie Validität und Reliabilität auch die unternehmensspezifi- schen Kompetenzanforderungen berücksichtigen muss.

Die Bildungsbedarfsanalyse ist aufgaben- und anforderungsorientiert und unterstützt Personalentwickler vor allem bei der Planung von Weiterbildungsangeboten wie etwa Trainings. Sie wird meist vor der Ausgestaltung des firmeninternen Weiterbildungskatalogs in Form einer Umfrage, die sich an Führungskräfte richtet, durchgeführt. Das Vorgehen bei Mitarbeiterbefragungen mit Bildungsschwerpunkten ist ähnlich, doch analysieren diese über die Kompetenzentwicklungsbedarfe hinaus auch die Organisation als Lern- und Transferumgebung (Simon und Treiblmaier 2007, S. 335).

\subsection{Intervention}

Die Intervention, im Rahmen derer die eigentliche Kompetenzentwicklung betrieben wird, erfolgt in unterschiedlichen Formaten (siehe Abschn. 3.2), die sich in ihrer Ausprägung im Hinblick auf den Materialitäts- und Individualisierungsgrad unterscheiden. Zur Kompetenzentwicklung in Unternehmen tragen formale, nicht formale sowie informale Lernformate (EC 2001, S. 17) bei. Unter einem formalen Lernformat wie beispielsweise einem Executive MBA-Programm wird eine von Bildungseinrichtungen organisierte Weiterbildung verstanden, die mit einem Zertifikat abschließt. Nichtformale Lernformate sind wie formale Lernformate strukturiert, indem beispielsweise Lernziele expliziert werden und einem Lehrplan gefolgt wird. Das Erlangen von formal anerkannten Qualifikationen wird mit solchen Lernformaten jedoch nicht verbunden. Informale Weiterbildung basiert auf täglichen Erfahrungen und findet im Lernprozess selbst unstrukturiert statt, jedoch kann informale Weiterbildung ebenfalls über Informations- und Kommunikationstechnologie (IKT) unterstützte Strukturen und Prozesse organisiert werden, wobei hier - wie beim Wissensmanagement - eine Personalisierungsstrategie von einer Kodifizierungsstrategie unterschieden wird (Maier und Hädrich 2001, S. 498). Je nach Formalisierungsgrad der Weiterbildung findet die Steuerung der Kompetenzentwicklung schwerpunktmäßig bei der Bildungseinrichtung oder eher im Kontext des Unternehmens statt. Ein KMS hat idealerweise Kompetenzentwicklungsmaßnahmen aller Formate $\mathrm{zu}$ berücksichtigen.

\subsection{Evaluation von Kompetenzentwicklung}

Bei der Evaluation der Intervention wird zwischen formativer (gestaltender) und summativer (messender) Evaluation unterschieden (Caffarella 2002, S. 225). Die formative Evaluation zielt darauf ab, die Intervention bereits positiv $\mathrm{zu}$ beeinflussen. Dabei wird mithilfe von Reflexionsinstrumenten, die vor bzw. während der Wissensvermittlung eingesetzt werden, versucht, die Erwartungshaltungen gegenüber der Maßnahme und ihre Ausgestaltung aufeinander abzustimmen. Dieser Ansatz lässt sich auf Lernverträge übertragen, mit der Einschränkung, dass diese einen höheren Grad der Formalisierung aufweisen. Unter einem Lernvertrag wird ein Übereinkommen zwischen einer lernenden Person und deren Führungskraft verstanden. Dabei werden Lernziele und Lernstrategie sowie die Möglichkeiten der Überprüfung der Lernergebnisse festgelegt (Knowles 1986, S. 38). Ähnlich der formativen Evaluation hat der Lernvertrag in der Intervention auf das Lernfeld eine direkt steuernde Wirkung.

Der Nachweis von Kompetenzentwicklungsfortschritten und daraus resultierende verbesserte Unternehmensergebnisse stellen einen wesentlichen Aspekt des Kompetenzmanagements dar (Heyse und Ortmann 2008, S. 14). Bei der summativen Evaluation von strukturierten Lernformaten hat das VierPhasen-Modell von Kirkpatrick einen hohen Bekanntheitsgrad erlangt, es unterscheidet zwischen Zufriedenheitsmessung (Reaktion), Lernergebnismessung (Wissen), Lerntransfermessung (Verhalten) und der ökonomischen Lernerfolgsmessung im Transferfeld (Resultat) (Kirkpatrick und Kirkpatrick 2005, S. 21-26). Die ökonomische Lernerfolgsmessung wurde in den letzten Jahren unter dem Titel „Return-on-TrainingInvestment"-Rechnung weiterentwickelt (Phillips 2003, S. 35).

\section{Prototypenentwicklung und Evaluation}

\subsection{Prototypenentwicklung}

Um entsprechende Gestaltungsmöglichkeiten auch auf der Ebene der Kompetenzmanagementsoftware vorzufinden, wurde ein Prototyp entwickelt. Tabelle 2 


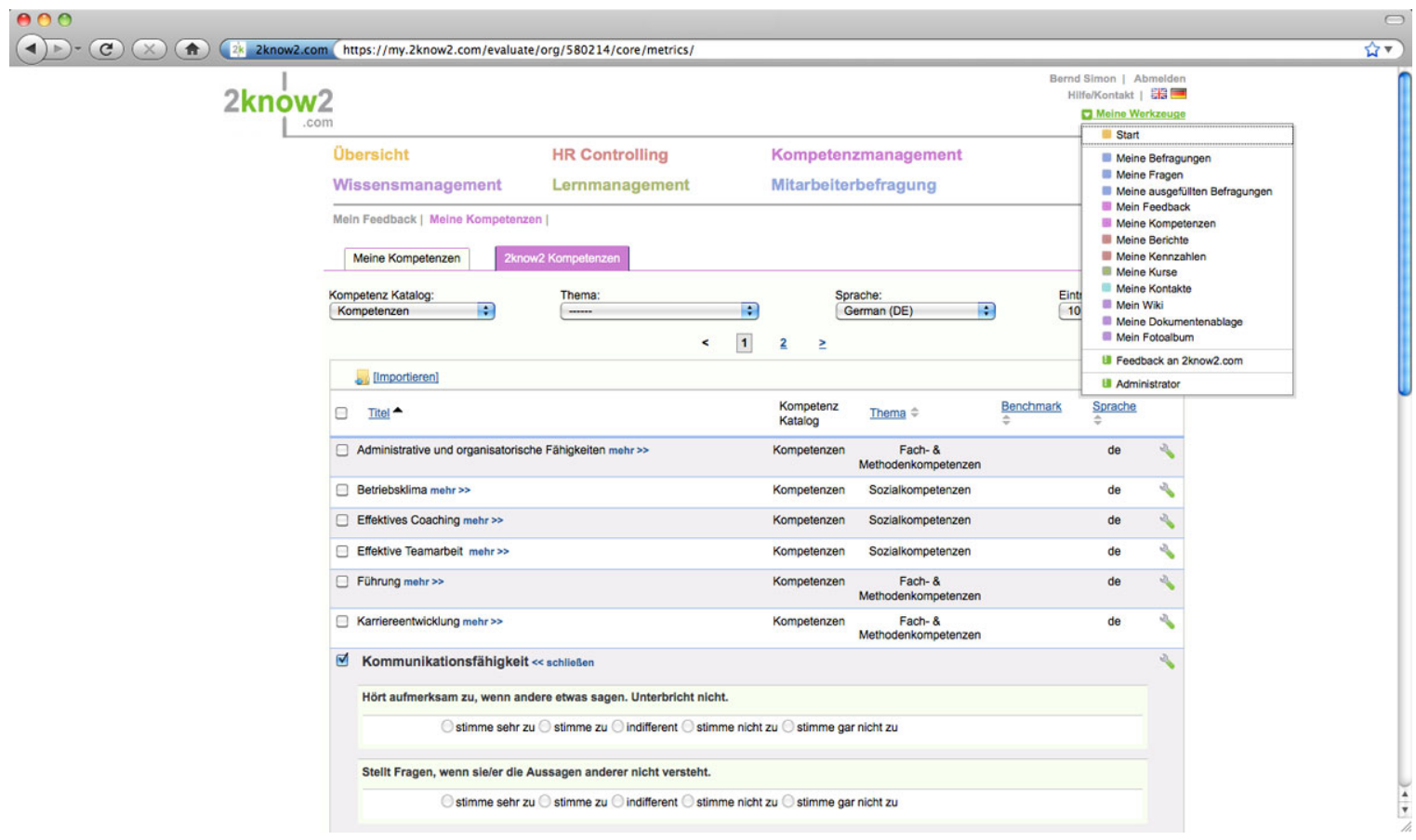

Abb. 1 Bildschirmfoto des organisationsübergreifenden Kompetenzkatalogs auf 2know2.com

Tab. 2 Am Prototyp implementierte Dienste

\begin{tabular}{lll}
\hline Grundanforderungen & Kompetenzmanagementdienste & Basisdienste \\
\hline Bedarfs- und Zielanalyse & 360-Grad-Feedback, & Benutzerverwaltung, \\
& Mitarbeitergespräch & Dateiablage, Kalender, Forum, \\
Intervention & Lernmanagement & Fotoalbum, Mail-Vorlagen, \\
Evaluation & Lernfortschrittskontrolle, & Musterfragen, Kennzahlen, \\
& Lerntransfermessung, & Kompetenzkatalog, \\
& Veranstaltungsevaluierung & \\
\hline
\end{tabular}

präsentiert die aus den Grundanforderungen (siehe Abschn. 4) abgeleiteten Kompetenzmanagementdienste. Diese orientieren sich an den oben angeführten Methoden und greifen auf $\mathrm{Ba}$ sisdienste zurück. Sie unterstützen Personalentwickler, Führungskräfte und externe Berater sowie Trainer in ihrer Arbeit, den Kompetenzbedarf in einem Unternehmen zu analysieren (z. B. mithilfe von 360-Grad-Feedback), Interventionen umzusetzen (z. B. mit Lernmanagement) und deren Erfolg zu evaluieren (z. B. mit Lerntransfermessung).

Der Prototyp stellt die Kompetenzmanagementdienste als ein für jedes Unternehmen flexibel konfigurierbares Portfolio von Softwarediensten bereit. Die Software-as-a-Service-Lösung erfordert keine Installation beim Kunden vor Ort und bietet unternehmensübergreifend ein Portfolio an wieder verwendbaren Inhalten an. Dazu zählen beispielsweise Fragenkataloge für Veranstaltungsevaluierungen und Bildungsumfragen, Kennzahlenkataloge für Leistungs- und Aktivitätsberichte von Personalabteilungen (sog. HRControlling) sowie Kompetenzkataloge. Abbildung 1 zeigt das Bildschirmfoto eines Kompetenzkatalogs, wie er beispielsweise in einem 360-Grad-Feedback Verwendung findet. Inzwischen wurde der Prototyp erfolgreich am Markt eingeführt und wird von 15 Organisationen (Stand: August 2010) verwendet.

\subsection{Evaluationsergebnisse}

Die mittels Inhaltsanalyse ausgewerteten Interviews führen $\mathrm{zu}$ Ergebnissen, die sich den vier Bestandteilen von KMS (siehe Abschn. 4) zuordnen lassen.

\subsubsection{Strukturen und Prozesse der Organisation}

Gemeinsame Definition der Zielsetzung: Die in die Kompetenzentwicklungsprozesse involvierten Personen sind vor Einführung des KMS zu identifizieren und sollten auf die Definition von Zielsetzung und Umsetzung der Prozesse Einfluss ausüben können. Von vielen Interviewpartnern wurde bestätigt, dass so ein Vorgehen wünschwert ist und dass darüber hinaus soziale Aspekte im Zusammenhang mit der Projektdefinition bereits in einer Frühphase den Erfolg bzw. Misserfolg eines KMS bestimmen.

Steuerung von Aufmerksamkeit: Bei der Steuerung von Kompetenzentwicklung handelt es sich um Prozesse, die parallel zu den Kerngeschäftsprozessen eines Unternehmens stattfinden. Einige der Befragten äußerten in diesem Zusammenhang den Wunsch, im KMS Möglichkeiten einzubinden, um die Aufmerksamkeit der involvierten Personen auf die Kompetenzentwicklung zu lenken. KMS, die der Steuerung von Aufmerksamkeit eine besondere Bedeutung beimessen, scheinen deshalb besonders erfolgversprechend.

Involvierende Reflexion der Daten: Viele der Befragten identifizierten die intensive, alle Akteure involvierende Reflexion der mittels Kompetenzmanagementsoft- 
ware gesammelten Daten als eine Schlüsselaktivität für die erfolgreiche Inbetriebnahme von KMS. Fälschlicherweise suggerieren die durch das KMS abgebildeten Automatismen an mancher Stelle eine bereits vollständige Betrachtung eines für die Kompetenzentwicklung relevanten Phänomens. In besonderen Fällen lässt sich jedoch nur mithilfe einer umfassenden Diskussion, die alle mit den Daten in Verbindung stehenden Akteure einbezieht, eine positive Veränderung realisieren.

Neben den Prozessen können auch andere für die Kompetenzentwicklung relevante Erfolgsfaktoren identifiziert werden, die der Organisation zuzuordnen sind.

Kompatibilität mit etablierter Kommunikationskultur: Unternehmen etablieren unterschiedliche Kommunikationskulturen, insbesondere im Hinblick auf den Einsatz elektronischer Medien. Wenige Male wurde in diesem Zusammenhang betont, dass die eingeführte Kompetenzmanagementsoftware sich als $\mathrm{zu}$ dieser Kultur kompatibel erweisen muss bzw. bei Bedarf entsprechende kulturverändernde Maßnahmen zu ergreifen sind.

Lernkultur: Bei der Einführung von KMS entstehen besondere Anforderungen an die Organisation, die neben prozessbedingten Umstellungen bestimmte Einstellungen im Umgang mit dem Thema Lernen erfordern. So konnte unternehmens- und anwendungsfallübergreifend klar herausgearbeitet werden, dass der Erfolg eines KMS stark mit der im Unternehmen vorherrschenden Lernkultur verknüpft ist. KMS etablieren sich dann erfolgreich in Unternehmen, wenn Fortbildung und Lernen am Arbeitsplatz ein zentraler Stellenwert eingeräumt wird und das Unternehmen als Organisation wahrgenommen wird, die bereit ist, in Lernen und Veränderung zu investieren. Auf organisatorischer Ebene müssen „motivationale" Voraussetzungen vorhanden sein (z. B. Streben nach Exzellenz, Kundenorientierung, Vermeidung von Fehlern) und gleichzeitig genügend Freiraum für die persönliche und organisatorische Weiterentwicklung bestehen.

Ausreichende Bereitstellung von Ressourcen: Ebenfalls unternehmens- und anwendungsfallübergreifend wurde betont, dass eine ausreichende Ressourcenausstattung für KMS-Projekte erfolgskritisch ist. Die besondere Herausforderung besteht hier in der Bereitstellung von internen Personalressourcen, die entsprechend steuernd auf das KMS-Projekt einwirken. Die Darstellung der mittelfristig realisierbaren Effizienz- und Effektivitätsverbesserungen kann helfen, eine entsprechende Ressourcenausstattung zu schaffen. In diesem Zusammenhang spielt das im Rahmen des KMS-Projekts eingebrachte Expertenwissen eine besondere Rolle, da es dazu beiträgt, die Effizienz- und Effektivitätsverbesserungen zu realisieren.

\subsubsection{Anwender und ihre Kompetenzen}

Methodenkompetenz: Die durch KMS unterstützten Aktivitäten wie etwa Mitarbeitergespräch, 360-Grad-Feedback oder Mitarbeiterbefragung erfordern eine spezifische Methodenkompetenz. Das dafür notwendige Wissen muss bei den Schlüsselmitarbeitern der jeweiligen Prozesse (z. B. Führungskraft, Personalentwickler) vorhanden sein, um einen erfolgreichen Einsatz zu gewährleisten, wie insbesondere die externen Weiterbildungsdienstleister betonten. Im KMS integrierte Lernmodule können hier Abhilfe schaffen.

Aktivierungsgrad der Akteure: Der Führungskraft kommt sowohl beim Umsetzen einer lernorientierten Unternehmenskultur als auch bei der operativen Unterstützung bei Einführung und Betrieb von KMS eine Schlüsselfunktion zu. Darüber hinaus sind Führungskräfte bei der operativen Lernsteuerung von wesentlicher Bedeutung, da sie bei der Definition von organisationsrelevanten Lernzielen über die Auswahl von Maßnahmen bis hin zur Evaluation entscheidende Beiträge zu leisten haben - dies meinten insbesondere interne Weiterbildungsdienstleister sowie Personalleiter. Umgekehrt meinen die Führungskräfte, dass es für diese Organisationsmitglieder wiederum erforderlich ist, sich in das Kerngeschäft zu involvieren und ein Verständnis für die dort vorherrschenden Herausforderungen zu entwickeln. Ebenso gilt es, die externen Weiterbildungsdienstleister (z. B. Trainer, WorkshopLeiter) in die Prozesse einzubinden und über Lernziele und Lernevaluation $\mathrm{zu}$ informieren. Aber auch den Lernenden kommt eine entscheidende Rolle bei der Umsetzung zu. Einstellungen wie Veränderungsbereitschaft oder ein Streben nach organisatorischen Verbesserungen im Arbeitsalltag tragen zur erfolgreichen Implementierung von KMS entscheidend bei.

\subsubsection{Verfügbare Input- und Steuerungsressourcen}

Inputressourcen: Ein KMS profitiert sehr stark von den bereitgestellten Inputressourcen wie Veranstaltungen, Trainer oder elektronischen Lerninhalten. Die Zufriedenheit der Teilnehmer mit diesem Angebot sowie der Umsetzungserfolg in der betrieblichen Praxis scheinen unternehmens- und anwendungsfallübergreifend höchst essenziell für den Erfolg von KMS. Bei einer solchen Erfolgsmessung kommen wiederum Steuerungsressourcen wie Vorlagen, Benchmark-Werte und Experten-Wissen zum Einsatz, die Kompetenzmanagement durch die Sammlung und Aufbereitung von Informationen unterstützen.

Adaptierbare Vorlagen: Die Bereitstellung von Vorlagen für Kennzahlen, Erhebungsinstrumente und Kompetenzkataloge beschleunigt die Einführung und den Betrieb von KMS. Ein qualitativ hochwertiger und umfangreicher sowie gut strukturierter Vorlagenkatalog scheint für einen schnellen Projektstart vor allem für mittelgroße Organisationen bzw. aus Sicht der Personalleiter besonders hilfreich. Die Vorlagen müssen dann so aufbereitet werden, dass sie selbsterklärend den Verwendungszweck darstellen. Eine tutorielle oder beratende Begleitung des Auswahlprozesses ist erwünscht.

Einige der Befragten meinten, dass der Mehrwert von Vorlagen erheblich reduziert wird, sollten diese nicht hinreichend anpassbar sein. Unternehmensstrategie und -kultur sowie interner Sprachgebrauch geben Anforderungen für die Adaption der eingesetzten Kennzahlen, Instrumente und Vorlagen vor, die es zu berücksichtigen gilt.

Benchmark-Werte: Werden zu den vorgegebenen Instrumenten, Kennzahlen und Kompetenzmodellen BenchmarkWerte angegeben, stellt dies für einige Unternehmen einen zusätzlichen Mehrwert dar. Besonders interessant sind interne Benchmarks für die Leistungsbewertung der Weiterbildungsdienstleister. Die Verwendung von externen Benchmark-Werten im eigenen Unternehmen setzt jedoch eine entsprechende Transparenz der Berechnung voraus, da die Daten der Benchmark-Partner zu den eigenen Daten passen müssen.

Unternehmensübergreifendes Expertenwissen: Aus der Forderung nach entsprechender Methodenkompetenz bei den Anwendern ließ sich vereinzelt darüber hinaus noch die Empfehlung ableiten, 
dass das KMS die Möglichkeit beinhalten sollte, Anwendern auch gleich die notwendigen Methodenkompetenzen zu vermitteln. Dies kann über vorgefertigte Lerninhalte (Kodifizierungsstrategie) oder durch Experten, die mit solchen Inhalten vertraut sind, erfolgen (Personalisierungsstrategie).

\subsubsection{Kompetenzmanagementsoftware}

Einfache Bedienbarkeit (Usability): Je einfacher die Kompetenzmanagementsoftware $\mathrm{zu}$ bedienen ist, desto eher sollte es gelingen, Akteure, die Kompetenzmanagement nicht zu ihren Kernaktivitäten zählen, zu involvieren. Die Interviews bestätigten an dieser Stelle häufig die üblichen Anforderungen an eine einfache Bedienbarkeit, wie etwa: Übersichtlichkeit (Aufgeräumtheit der Benutzeroberfläche), transparente Navigationsstrukturen oder ansprechende optische Gestaltung.

Anpassbare Funktionalität: Trotz eines hohen Grads an wahrgenommener „Standardisierung“ der verschiedenen Kompetenzmanagementmethoden stellt die Anpassbarkeit der Funktionalität für viele Befragte eine wesentliche Softwareeigenschaft dar. Umfragen werden zum Beispiel wahlweise anonym, personenbezogen oder mithilfe anonymisierter Kennungen durchgeführt. Veranstaltungen erfordern alternativ die Integration von Teilnahmebedingungen oder etwa die Freigabe einer oder mehrerer Führungskräfte. Die Definition von FeedbackProzessen kann durch die Personalleiter, durch externe Berater oder durch die Feedbackempfänger selbst vorgenommen werden. Kompetenzmanagementsoftware muss deshalb entsprechend flexibel konfigurierbar sein, so einige der Befragten.

Interoperabilität: Der Betrieb einer Kompetenzmanagementsoftware erfordert eine Reihe von Schnittstellen zu anderen inner- und außerbetrieblichen Informationssystemen. Diese Schnittstellen dienen bei den Stammdaten der Vermeidung von Datenredundanzen und den damit verknüpften Effizienzsteigerungen. Andererseits sind Schnittstellen für die Weiterbearbeitung der gesammelten Informationen und die Zusammenarbeit rund um diese von essenzieller Bedeutung und tragen so zu einem effektiveren KMS bei. Obwohl keine IKTSpezialisten befragt wurden, wurde die Anforderung „Interoperabilität“ relativ oft genannt.
Transparenz von Kompetenzentwicklungsprozessen und Ergebnissen: Als großer Vorteil wurde in einigen wenigen Fällen die mit KMS neu geschaffene Transparenz genannt. Die in der Kompetenzmanagementsoftware verfügbaren Daten flexibel und umfassend auswerten zu können, stellt einen Schlüsselfaktor für eine verbesserte Steuerung der Kompetenzentwicklung dar. Deshalb ist es wesentlich, dass - unter Berücksichtigung personalrechtlicher Aspekte - Akteure Zugang zu den entsprechenden Steuerungsinformationen erhalten. Die einfache Gewinnung und Abrufbarkeit von für die Kompetenzentwicklung relevanten Informationen wie zum Beispiel bestehender Kompetenzen und Kompetenzentwicklungsziele, wahrgenommener Lerntransfer, Veranstaltungsqualität oder Umsetzung der Personalführungsrolle schaffen neue Möglichkeiten für Effektivitätssteigerungen. Meta-Steuerungsinformationen wie beispielsweise die Anzahl der ausgefüllten Fragebogen oder der durchgeführten Mitarbeitergespräche schaffen zusätzliche Transparenz hinsichtlich der Implementierung der für das KMS relevanten Prozesse.

Schutz der Privatsphäre: Die in Kompetenzmanagementsoftware verfügbaren personenbezogenen Daten wie Personalstammdaten, Kompetenzprofile oder absolvierte Weiterbildungen werden naturgemäß als sehr persönliche und schützenswerte Daten empfunden. D. h., Anwender haben ein großes Interesse, die Sichtbarkeit dieser Daten steuern zu können. So meinten etwa einige Personalleiter, dass selbst eine über eine $\mathrm{Ab}$ teilungssicht hinausgehende Transparenz von Weiterbildungsaktivitäten missfallen kann. Gleichzeitig gilt es auch bei der Datenerhebung, insbesondere bei Umfragen, die Vertraulichkeit der behandelten Daten sicherzustellen und beispielsweise anonyme Antwortmöglichkeiten $\mathrm{zu}$ schaffen. Viele der Befragten meinten, dass die Dialoge zur Benutzerführung gewährleisten müssen, dass die Kompetenzmanagementsoftware als vertrauenswürdiges und die eigene Privatsphäre schützendes Medium empfunden wird.

\section{Zusammenfassung und Implikationen für die Forschung}

Die vorliegende Arbeit stellt Gestaltungsprinzipien für KMS vor, indem zuerst relevante Schlüsselbegriffe, begründende
Theorien und Grundanforderungen präsentiert werden. Auf Basis einer prototypgetriebenen Evaluation mit anschlieBender Inhaltsanalyse der Interviewtranskriptionen konnte eine Reihe von Gestaltungsempfehlungen (sog. Propositionen) identifiziert werden, um eine personenzentrierte Entwicklung von Kernkompetenzen zu unterstützen. Die entwickelten Propositionen sind als theorienentwickelnd (vs. theorienprüfend) $\mathrm{zu}$ verstehen (Atteslander 2008, S. 200). Um die Allgemeingültigkeit der Propositionen $\mathrm{zu}$ überprüfen, wäre eine modellhafte Zusammenführung dieser Empfehlungen umzusetzen und $\mathrm{zu}$ testen. Die auf Basis solcher Effektivitätsmodelle durchgeführten, quantitativen Untersuchungen würden zusätzliche Erkenntnisse hinsichtlich der unterschiedlichen Bedeutung der einzelnen Gestaltungsempfehlungen ermöglichen. Davor gilt es jedoch noch die identifizierten Gestaltungsprinzipien noch weiter zu vertiefen und Unterschiede für verschiedene Anwendungsbereiche und Anwendertypen herauszuarbeiten.

Eine Weiterentwicklung der Untersuchung, die das Talentmanagement in den Blick nähme, wobei hier neue Funktionen zur Rekrutierung, Talentidentifizierung, Leistungsbeurteilung und Nachfolgeplanung zu behandeln sind (Steinweg 2009, S. 8), wäre eine wertvolle Fortführung dieses Forschungsprojekts.

Als Limitation des derzeitigen Forschungsstandes ist zu erwähnen, dass eine kompetenzorientierte Steuerung von Lernaktivitäten, wie sie auch diesem Beitrag zu Grunde liegt, letztendlich nur eine von vielen etablierten IKT-unterstützten, personenbezogenen Praktiken darstellt, die auf die Unternehmensleistung Einfluss nehmen. In der betriebswirtschaftlichen Literatur finden sich darüber hinaus kontroll-, „commitment“- sowie remunerationsorientierte Führungstheorien (Sonntag 2005), die es als theoretische Basis von KMS noch zu untersuchen gilt.

Die Studie wurde vor allem im Kontext von Kompetenzentwicklungsmaßnahmen durchgeführt, die auf formalen Steuerungsmethoden sowie auf formalen und nichtformalen Lernformate beruhten. Eine Erweiterung um informale Lernformate könnte neue, zusätzliche Erkenntnisse liefern und die Lücke zwischen Wissensmanagement und Kompetenzmanagement weiter schließen. 


\section{Danksagung}

Die Arbeit wurde mit Fördermitteln des 6. Rahmenprogramms der Europäischen Kommission unterstützt (Forschungsprojekt „PROLIX - Process-oriented Learning and Information eXchange“, Vertragsnummer: 027905). Der Autor möchte darüber hinaus am Forschungsprojekt beteiligten Kollegen und Studierenden am Lehrstuhl „Wirtschaftsinformatik und Neue Medien“ an der WU sowie den Entwicklern und Beratern der „Knowledge Markets“ Consulting Ges.m.b.H. in Wien für ihre unzähligen Beiträge danken.

Open Access Dieser Artikel unterliegt den Bedingungen der Creative Commons Attribution Noncommercial License. Dadurch sind die nichtkommerzielle Nutzung, Verteilung und Reproduktion erlaubt, sofern der/die Originalautor/en und die Quelle angegeben sind.

\section{Zusammenfassung}

Bernd Simon

\section{Gestaltungstheoretische Überlegungen zu Kompetenzmanagementsystemen}

Die Kompetenzen ihrer Mitarbeiter stellen für jede Organisation einen kritischen Erfolgsfaktor dar. Kompetenzmanagementsysteme helfen dabei, Kompetenzen zu entwickeln, indem Prozesse wie Bedarfsanalyse, Intervention und Evaluation unterstützt werden. Bei der Gestaltung von Kompetenzmanagementsystemen treffen Organisationen jedoch auf eine Vielzahl technischer und organisatorischer Herausforderungen. Dieser Beitrag adressiert diese Herausforderungen und präsentiert gestaltungstheoretische Überlegungen für die Entwicklung von Kompetenzmanagementsystemen. Die im Rahmen einer Aktionsforschung durchgeführte Prototypenevaluation involvierte 15 Unternehmen und identifizierte Gestaltungsempfehlungen in den Bereichen Organisation, Anwender, Ressourcen und Kompetenzmanagementsoftware.

Schlüsselwörter: Kompetenzmanagementsystem, Ressourcenbasierte Führungstheorie, Personalentwicklung, Lernmanagement, Designwissenschaften, Prototypenevaluation, Aktionsforschung

\section{A Discussion on Competency Management Systems from a Design Theory Perspective}

The competencies of employees are a critical success factor for any organization. Competency Management Systems help to develop competencies by supporting processes such as needs identification, intervention planning and implementation, and evaluation. However, the design of Competency Management Systems comes with a broad range of technical and organizational challenges. This study addresses these challenges and discusses the development of Competency Management Systems from a design theory perspective. The prototype evaluation, conducted as part of an action research initiative, involved fifteen companies and identified design recommendations in the areas of organization, user, resources, and competency management software.

Keywords: Competency Management Systems, Resource-based view of the firm, Human resource development, Learning management, Design sciences, Prototype evaluation, Action research 


\section{Anhang: Inhaltsanalyse am Beispiel der Gestaltungsempfehlung „Benchmark-Werte“}

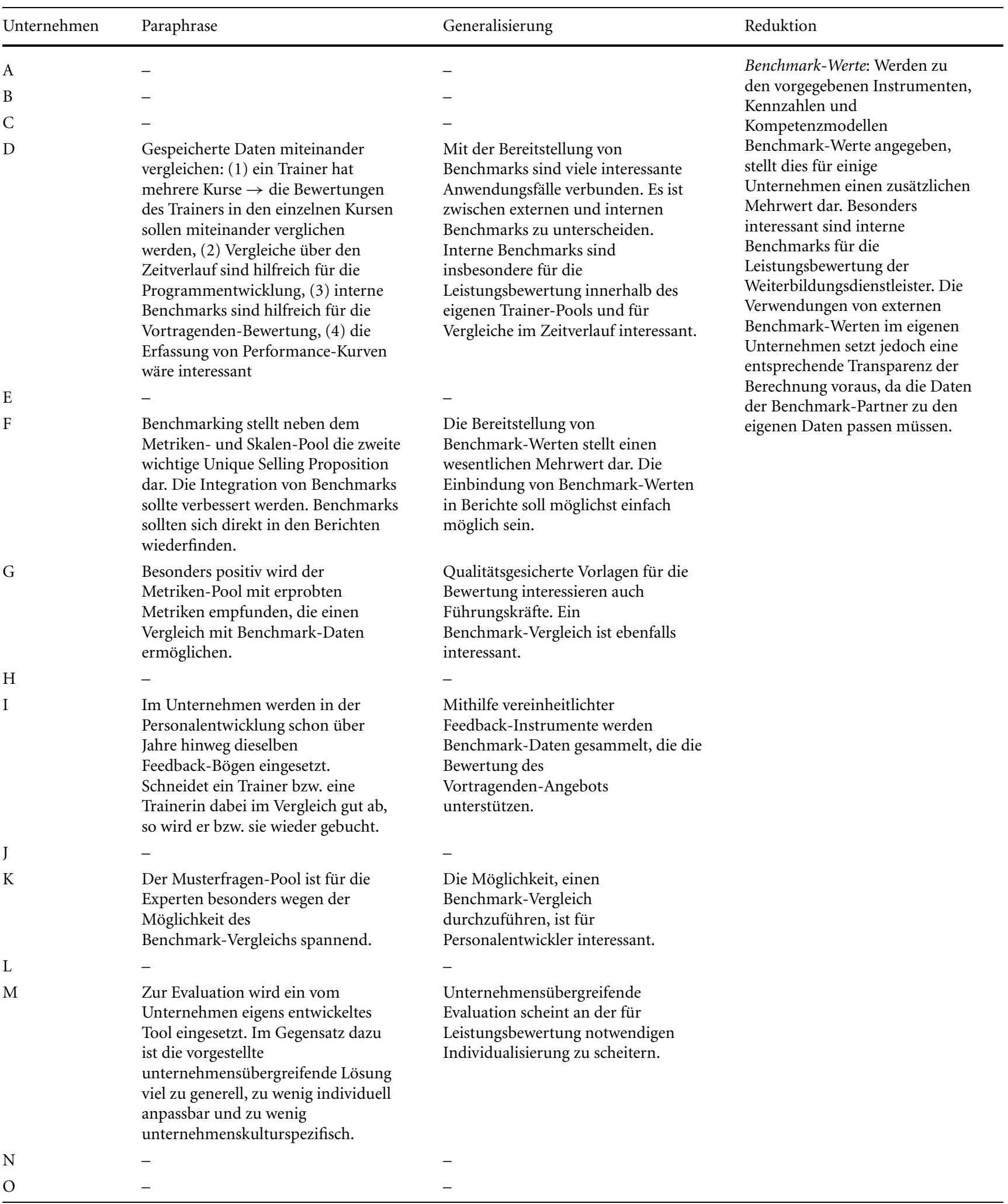




\section{Literatur}

Argote L, McEvily B, Reagans R (2003) Managing knowledge in organizations: an integrative framework and review of emerging themes. Management Science 49(4):571582

Atteslander P (2008) Methoden der empirischen Sozialforschung. Erich Schmidt, Berlin

Baskerville R, Wood-Harper AT (1998) Diversity in information systems action research methods. European Journal of Information Systems 7(2):90-107

Caffarella RS (2002) Planning programs for adult learners - a pratical guide for educators, trainers, and staff developers. Wiley, San Francisco

Czerny E (2009) Nachhaltiges Kompetenzmanagement: mit den Kompetenzen der Mitarbeiter zu dauerhaftem Unternehmenserfolg; eine ressourcenorientierte Betrachtung. Müller, Saarbrücken

Davis FD (1989) Perceived usefulness, perceived ease of use and user acceptance of information technology. MIS Quarterly 13(3):319-339

Davison RM, Martinsons MG, Kock G (2004) Principles of canonical action research. Information Systems Journal 14:65-86

Deiser R (2009) Designing the smart organization - how breakthrough corporate learning initiatives drive strategic change and innovation. Jossey-Bass, San Francisco

DeLone WH, McLean ER (2003) The DeLone and McLean model of information systems success: a ten-year update. Journal of Management Information Systems 19(4):9-30

Devaraj S Babu SR (2004) How to measure the relationship between training and job performance. Communications of the ACM 47(5):63-67

Easterby-Smith $M$, Thorpe R, Jackson PR (2008) Management research. Sage, London

EC (2001) Making a European area of lifelong learning a reality. European Commission, Brussels

Edwards MR, Ewen AJ (2000) 360 Beurteilung - Klareres Feedback, höhere Motivation und mehr Erfolg für alle Mitarbeiter. Beck, München

Engelhart W, Kleinaltenkamp M, Rechenfelderbäumer M (1993) Leistungsbündel als Absatzobjekte - Ein Ansatz zur Überwindung der Dichotomie von Sach- und Dienstleistungen. zfbf - Zeitschrift für betriebswirtschaftliche Forschung 45(5):395426

Erpenbeck J, Sauter W (2007) Kompetenzentwicklung im Netz - New Blended Learning mit Web 2.0. Luchterhand, Köln
Faltin N, Zimmermann V (2009) Nutzloses Training adé - Prozessorientierte Lerntechnologien für kompetente Mitarbeiter. Information Management \& Consulting 24(1):614

Gregor S, Jones D (2007) The anatomy of a design theory. Journal of the Association for Information Systems 8(5):312-355

Hamel G (2007) The future of management. Harvard Business School Press, Boston

Hasenkamp U, Stahlknecht P (2009) Das Fach Wirtschaftsinformatik im Spiegel seiner Zeitschrift. WIRTSCHAFTSINFORMATIK 51(1):18-27

Hertel G, Konradt U (2004) Human Resource Management im Inter- und Intranet: Inhalte und Überblick. In: Hertel G, Konradt U (Hrsg) Human Resource Management im Inter- und Intranet. Hogrefe, Göttingen

Hevner AR, March ST Park J (2004) Design research in information systems research. MIS Quarterly 20(1):75-105

Heyse V, Ortmann S (2008) Talentmanagement in der Praxis: Eine Anleitung mit Arbeitsblättern, Checklisten, Softwarelösungen. Waxmann, Münster

Kirkpatrick DL, Kirkpatrick JD (2005) Evaluating training programs: the four levels. Berrett-Koehler, Berkley

Knowles MS (1986) Using learning contracts practical approaches to individualizing and structuring learning. Jossey-Bass, San Francisco

Laudon KC, Laudon JP (2005) Essentials of management information systems. Pearson Education, Upper Saddle River

Lindgren R, Henfridsson O, Schultze U (2004) Design principles for competence management systems: a synthesis of an action research study. MIS Quarterly 28(3):435-472

Macharzina K, Wolf J (2008) Unternehmensführung. Gabler, Wiesbaden

Maier R, Hädrich T (2001) Modell für die Erfolgsmessung von Wissensmanagementsystemen. WIRTSCHAFTSINFORMATIK 43(5):497-509

Markus ML, Majchrzak A, Gasser L (2002) A design theory for systems that support emergent knowledge processes. MIS Quarterly 26(3):179-212

Mayring P (2010) Qualitative Inhaltsanalyse - Grundlagen und Techniken. Beltz, Weinheim

Patton MQ (2002) Qualitative research \& evaluation methods. Sage, Thousand Oaks

Phillips JJ (2003) Return on investment in training and performance improvement programs. Butterworth \& Heinemann, Amsterdam

Picot A, Baumann O (2009) Die Bedeutung der Organisationstheorie für die Ent- wicklung der Wirtschaftsinformatik. WIRTSCHAFTSINFORMATIK 51(1):72-81

Prahalad CK, Hamel G (1990) The core competence of the corporation. Harvard Business Review 68(3):79-91

Probst G, Raub S, Romhardt K (2000) Managing knowledge. Building blocks for success. Wiley, Chichester

Rosenstiel LV (2006) Entwicklung von Werthaltungen und individueller Kompetenz. In: Sonntag K (Hrsg) Personalentwicklung in Organisationen. Hogrefe, Göttingen

Rubin HJ, Rubin IS (1995) Qualitative Interviewing. Sage, Thousand Oaks

Schuler H, Görlich Y. (2006) Ermittlung erfolgsrelevanter Merkmale von Mitarbeitern. In: Sonntag K (Hrsg) Personalentwicklung in Organisationen. Hogrefe, Göttingen

Seeber S (2000) Stand und Perspektiven von Bildungscontrolling. In: Seeber S, Krekel E, Van Buer J (Hrsg) Bildungscontrolling: Ansätze und kritische Diskussion zur Effizienzsteigerung von Bildungsarbeit. Lang, Frankfurt am Main

Simon B, Ostertag R, Machal R, Weihs $\mathrm{P}$ (2008) Qualifizierung von Lieferanten über eLearning - Ein Pilotprojekt der Daimler AG. HMD - Praxis der Wirtschaftsinformatik 259:96-104

Simon B, Treiblmaier H (2007) Von der Qualitätssicherung zum Bildungscontrolling: Fortbildungsmanagement unter der Effektivitätslupe. In: Breitner MH, Bruns B, Lehner $F$ (Hrsg) Neue Trends im E-Learning: Aspekte der Betriebswirtschaftslehre und Informatik. Physica-Verlag, Heidelberg

Solga M, Ryschka J, Mattenklott A (2008) Personalentwicklung: Gegenstand, Prozessmodell, Erfolgsfaktoren. In: Ryschka J, Solga M, Mattenklott A (Hrsg) Praxishandbuch Personalentwicklung - Instrumente, Konzepte, Beispiele. GMW, Wiesbaden

Sonntag K (2005) Personalentwicklung in Organisationen. Hogrefe, Göttingen

Steinweg S (2009) Systematisches Talent Management: Kompetenzen strategisch einsetzen. Schäffer-Poeschel, Stuttgart

Strohmeier S (2000) Informatisierung der Personalwirtschaft: Eine kritische Bestandsaufnahme gegenwärtiger Forschung. WIRTSCHAFTSINFORMATIK 42(Sonderheft):9096

Venkatesh V (2000) Determinants of perceived ease of use: integrating control intrinsic motivation, and emotion into the technology acceptance model. Information Systems Research 11(4):342-365

Zwick T (2004) Weiterbildungsintensität und betriebliche Produktivität. Zeitschrift für Betriebswirtschaft 74(7):651-668 